\title{
A conjecture of Cameron and Kiyota on sharp characters with prescribed values
}

\author{
A. Abdollahi ${ }^{a, c}$, J. Bagheriand1, M. Khatami $^{a}$, Z. Shahbazi $^{a}$, R. Sobhani ${ }^{b}$ \\ ${ }^{a}$ Department of Pure Mathematics, Faculty of Mathematics and Statistics, University of \\ Isfahan, Isfahan 81746-73441, Iran \\ ${ }^{b}$ Department of Applied Mathematics and Computer Science, Faculty of Mathematics \\ and Statistics, University of Isfahan, Isfahan 81746-73441, Iran \\ ${ }^{c}$ School of Mathematics, Institute for Research in Fundamental Sciences (IPM),
} 19395-5746 Tehran, Iran

Emails: a.abdollahi@sci.ui.ac.ir, bagherian@sci.ui.ac.ir, m.khatami@sci.ui.ac.ir, z.shahbazi@sci.ui.ac.ir, r.sobhani@sci.ui.ac.ir

\begin{abstract}
Let $\chi$ be a virtual (generalized) character of a finite group $G$ and $L=L(\chi)$ be the image of $\chi$ on $G-\{1\}$. The pair $(G, \chi)$ is said to be sharp of type $L$ if $|G|=\prod_{l \in L}(\chi(1)-l)$. If the principal character of $G$ is not an irreducible constituent of $\chi$, the pair $(G, \chi)$ is called normalized. In this paper, we first provide some counterexamples to a conjecture that was proposed by Cameron and Kiyota in 1988. This conjecture states that if $(G, \chi)$ is sharp and $|L| \geq 2$, then the inner product $(\chi, \chi)_{G}$ is uniquely determined by $L$. We then prove that this conjecture is true in the case that $(G, \chi)$ is normalized, $\chi$ is a character of $G$, and $L$ contains at least an irrational value.
\end{abstract}

Keywords: Character value; Finite group; Sharp character; Sharp pair.

MSC(2010): 20C15.

\section{Introduction}

Let $G$ be a finite group and $\chi$ be a virtual (generalized) character of $G$. We define

$$
L(\chi):=\{\chi(g) \mid 1 \neq g \in G\}
$$

and

$$
S h(\chi)=\prod_{l \in L(\chi)}(\chi(1)-l) .
$$

\footnotetext{
${ }^{1}$ Corresponding author
} 
It is known that $S h(\chi)$ is a multiple of $|G|$; see [2]. The pair $(G, \chi)$ (or briefly, the virtual character $\chi)$ is called sharp of type $L$ or $L$-sharp if $L=L(\chi)$ and $S h(\chi)=|G|$. Clearly, $\chi$ is faithful whenever $(G, \chi)$ is $L$-sharp. The pair $(G, \chi)$ is said to be normalized if $\left(\chi, 1_{G}\right)_{G}=0$, where $1_{G}$ is the principal character of $G$.

The notion of sharpness was first introduced for permutation characters by Ito and Kiyota as a generalization of sharply $t$-transitive permutation representations [4]. Then, Cameron and Kiyota extended the definition of sharp pairs to what is given above and posed the following conjecture [3].

Conjecture 1: If $(G, \chi)$ is $L$-sharp with $|L| \geqslant 2$, then $(\chi, \chi)_{G}$ is uniquely determined by $L$.

As a main result in this paper, we provide some counterexamples to the above conjecture. In Section 2, we give a group $G$ and two characters $\chi$ and $\theta$ of $G$ such that the pairs $(G, \chi)$ and $(G, \theta)$ are $L$-sharp, but $(\chi, \chi)_{G} \neq(\theta, \theta)_{G}$ (see Example 2.1). Moreover, we show that if $(G, \chi)$ in Conjecture 1 is assumed to be normalized, then there are some counterexamples which show $(\chi, \chi)_{G}$ is not uniquely determined by $L$ (see Example 2.2). It should be emphasized that we can not find any counterexample to Conjecture 1 in the case that $(G, \chi)$ is normalized and $\chi$ is a character. It seems that this conjecture is true whenever $(G, \chi)$ is normalized and $\chi$ is a character. Therefore, we give an improvement of Conjecture 1 as follows.

Conjecture 2: If $(G, \chi)$ is $L$-sharp and normalized where $\chi$ is a character and $|L| \geqslant 2$, then $(\chi, \chi)_{G}$ is uniquely determined by $L$.

In Section 3, we show that Conjecture 2 is true whenever $L$ contains at least an irrational value. To do this, we use a characterization of $L$-sharp pairs, given in [1], when $L$ contains at least an irrational value.

\section{L-Sharp characters with different numbers of irreducible constituents}

In this section, we first give a group $G$ with two characters $\chi$ and $\theta$ such that $(G, \chi)$ and $(G, \theta)$ are $L$-sharp and $(\chi, \chi)_{G} \neq(\theta, \theta)_{G}$ (see Example 2.1). This shows that 
Conjecture 1 is not true, in general.

Example 2.1. Let $G$ be the group $\operatorname{SmallGroup}(32,6)$ in GAP [5]. Put $\chi=\chi_{1}+$ $2 \chi_{2}+\chi_{5}$. Then as we can see in Table $1, \chi$ is sharp of type $L=\{-1,3\}$ and $(\chi, \chi)_{G}=6$. On the other hand, set $\theta=\chi_{2}+\chi_{3}+\chi_{4}+\chi_{5}$. Then $\theta$ is also sharp of type $L=\{-1,3\}$ such that $(\theta, \theta)_{G}=4$. But we have $(\chi, \chi)_{G} \neq(\theta, \theta)_{G}$.

\begin{tabular}{c|rrrrrrrrrrr}
$\left|C l_{G}(g)\right|$ & 1 & 4 & 4 & 2 & 2 & 1 & 4 & 4 & 4 & 2 & 4 \\
\hline$\chi_{1}$ & 1 & 1 & 1 & 1 & 1 & 1 & 1 & 1 & 1 & 1 & 1 \\
$\chi_{2}$ & 1 & -1 & 1 & 1 & 1 & 1 & -1 & -1 & 1 & 1 & -1 \\
$\chi_{3}$ & 1 & 1 & -1 & 1 & 1 & 1 & -1 & 1 & -1 & 1 & -1 \\
$\chi_{4}$ & 1 & -1 & -1 & 1 & 1 & 1 & 1 & -1 & -1 & 1 & 1 \\
$\chi_{5}$ & 4 & 0 & 0 & 0 & 0 & -4 & 0 & 0 & 0 & 0 & 0
\end{tabular}

Table 1: Sharp characters $\chi=\chi_{1}+2 \chi_{2}+\chi_{5}$ and $\theta=\chi_{2}+\chi_{3}+\chi_{4}+\chi_{5}$

The example below shows that Conjecture 1 is not true if we further assume that $(G, \chi)$ is normalized.

Example 2.2. Let $G$ be the group SmallGroup $(192,1494)$ in GAP [5]. Put $\chi=$ $\chi_{2}+\chi_{5}$. Then $\chi$ is sharp of type $L=\{-2,0,2\}$ and $(\chi, \chi)_{G}=2$; see Table 2. On the other hand, set $\theta=\chi_{3}+\chi_{4}-\chi_{1}$. Then $\theta$ is also sharp of type $L=\{-2,0,2\}$ such that $(\theta, \theta)_{G}=3$. Clearly, $(\chi, \chi)_{G} \neq(\theta, \theta)_{G}$.

\begin{tabular}{c|rrrrrrrrrrrrr}
$\left|C l_{G}(g)\right|$ & 1 & 24 & 32 & 6 & 6 & 1 & 12 & 24 & 32 & 12 & 6 & 24 & 12 \\
\hline$\chi_{1}$ & 1 & -1 & 1 & 1 & 1 & 1 & -1 & -1 & 1 & 1 & 1 & -1 & -1 \\
$\chi_{2}$ & 2 & 0 & -1 & 2 & 2 & 2 & 0 & 0 & -1 & 2 & 2 & 0 & 0 \\
$\chi_{3}$ & 3 & 1 & 0 & -1 & -1 & 3 & -1 & -1 & 0 & -1 & 3 & 1 & -1 \\
$\chi_{4}$ & 4 & 0 & 1 & 0 & 0 & -4 & -2 & 0 & -1 & 0 & 0 & 0 & 2 \\
$\chi_{5}$ & 4 & 0 & 1 & 0 & 0 & -4 & 2 & 0 & -1 & 0 & 0 & 0 & -2
\end{tabular}

Table 2: Sharp characters $\chi=\chi_{2}+\chi_{5}$ and $\theta=\chi_{3}+\chi_{4}-\chi_{1}$

In the following, we give some remarks related to Conjecture 2.

Remark 2.3. We can express a strong version of Conjecture 2 as follows:

If $(G, \chi)$ and $(H, \theta)$ are L-sharp and normalized such that $\chi$ and $\theta$ are characters and $|L| \geqslant 2$, then $(\chi, \chi)_{G}=(\theta, \theta)_{H}$. 
By Example 2.4 below, we show that the above conjecture is not true, in general. In fact, there exist non-isomorphic groups $G$ and $H$ with $L$-sharp characters $\chi$ and $\theta$ with $|L| \geq 2$ such that $(\chi, \chi)_{G} \neq(\theta, \theta)_{H}$. Moreover, if we further assume that $|G|=|H|$, then there exists an counterexample to the above conjecture; see Example 2.5.

Example 2.4. Let $G$ be the alternating group $A_{7}$ of degree 7. Using the character table of $G$, one can check that $G$ has an irreducible sharp character $\chi$ of type $L=\{-1,0,2\}$; see Table 3 . On the other hand, let $H$ be the dihedral group $D_{12}$ of order 12. Then $H$ has the sharp character $\theta=\chi_{2}+\chi_{6}$ of type $L=\{-1,0,2\}$; see Table 4. Clearly, in this case $(\chi, \chi)_{G} \neq(\theta, \theta)_{H}$.

\begin{tabular}{c|ccccccccc}
$\left|C l_{G}(g)\right|$ & 1 & 105 & 70 & 210 & 280 & 630 & 504 & 360 & 360 \\
\hline$\chi$ & 14 & 2 & 2 & 2 & -1 & 0 & -1 & 0 & 0
\end{tabular}

Table 3: Sharp character $\chi$

\begin{tabular}{c|rrrrrr}
$\left|C l_{G}(g)\right|$ & 1 & 1 & 3 & 3 & 2 & 2 \\
\hline$\chi_{1}$ & 1 & 1 & 1 & 1 & 1 & 1 \\
$\chi_{2}$ & 1 & 1 & -1 & -1 & 1 & 1 \\
$\chi_{3}$ & 1 & -1 & -1 & 1 & 1 & -1 \\
$\chi_{4}$ & 1 & -1 & 1 & -1 & 1 & -1 \\
$\chi_{5}$ & 2 & 2 & 0 & 0 & -1 & -1 \\
$\chi_{6}$ & 2 & -2 & 0 & 0 & -1 & 1
\end{tabular}

Table 4: Character table of $D_{12}$

Example 2.5. Let $G$ be the group SmallGroup $(192,955)$ in GAP [5]. Then as we can see in Table 5, $G$ has an irreducible sharp character $\chi$ of type $L=\{-2,0,2\}$. On the other hand, let $H$ be the group SmallGroup $(192,1494)$ in GAP [5]. Using

\begin{tabular}{c|rrrrrrrrrrrrrr}
$\left|C l_{G}(g)\right|$ & 1 & 12 & 4 & 32 & 6 & 3 & 12 & 24 & 12 & 32 & 12 & 6 & 24 & 12 \\
\hline$\chi$ & 6 & -2 & 0 & 0 & 2 & -2 & 0 & 0 & 2 & 0 & 0 & -2 & 0 & 0
\end{tabular}

Table 5: Sharp character $\chi$

the character table of $H, \theta=\theta_{1}+\theta_{2}$ is a sharp character of type $L=\{-2,0,2\}$ (see Table 6). Clearly, in this case $(\chi, \chi)_{G} \neq(\theta, \theta)_{H}$. 


\begin{tabular}{c|rrrrrrrrrrrrr}
$\left|C l_{H}(h)\right|$ & 1 & 24 & 32 & 6 & 6 & 1 & 12 & 24 & 32 & 12 & 6 & 24 & 12 \\
\hline$\theta_{1}$ & 2 & 0 & -1 & 2 & 2 & 2 & 0 & 0 & -1 & 2 & 2 & 0 & 0 \\
$\theta_{2}$ & 4 & 0 & 1 & 0 & 0 & -4 & 2 & 0 & -1 & 0 & 0 & 0 & -2
\end{tabular}

Table 6: Sharp character $\theta=\theta_{1}+\theta_{2}$

\section{Sharp characters with at least an irrational value}

In this section, we show that if $(G, \chi)$ is $L$-sharp and normalized such that $\chi$ is a character and $L$ contains at least an irrational value, then Conjecture 2 holds. Our key tool is the following result given by Alvis and Nozawa in [1].

For the remainder of this paper, whenever $(G, \chi)$ is sharp, we assume that $\chi$ is a character of $G$.

Theorem 3.1. ([1, Theorem 1.3]) Suppose $(G, \chi)$ is L-sharp and normalized such that $L$ contains at least an irrational value. Then one of the following holds.

(i) $G$ is cyclic of order $m$, and either $m \geq 3$ and $\chi$ is linear, or else $m \geq 5$ and $\chi$ is the sum of two complex conjugate linear characters of $G$.

(ii) $G$ is dihedral of order $2 m$, where $m \geq 5$ is odd, and $\chi$ is irreducible of degree 2.

(iii) $G$ is dihedral or generalized quaternion of order $2 m$, where $m \geq 8$ is even, and $\chi=\psi$ or $\chi=\psi+\varepsilon$, where $\psi$ is irreducible of degree 2 and $\varepsilon$ is linear with cyclic kernel of order $m$.

(iv) $G$ is isomorphic to the binary octahedral group and $\chi$ is irreducible of degree 2 .

(v) $G$ is isomorphic to $S L(2,5)$ and $\chi$ is irreducible of degree 2.

(vi) $G$ is isomorphic to $A_{5}$ and $\chi$ is irreducible of degree 3.

The next lemmas give some results about the sharp characters of cyclic groups, dihedral groups, and generalized quaternion groups which we need in the sequel. 
Lemma 3.2. ([3, Proposition 1.8]) Let $G$ be a cyclic group of order $m$ and $\lambda$ be a faithful linear character of $G$. Then $(G, \lambda)$ is sharp of type

$$
L=\left\{\omega^{r} \mid 1 \leq r \leq m-1\right\}
$$

where $\omega=e^{2 \pi i / m}$.

Lemma 3.3. Let $G$ be a cyclic group of order $m$, where $m \geq 5$ is odd, and let $\lambda$ be a faithful linear character of $G$. Then $(G, \lambda+\bar{\lambda})$ is a sharp pair of type $L=$ $\left\{\omega^{r}+\omega^{-r} \mid 1 \leq r \leq(m-1) / 2\right\}$, where $\omega=e^{2 \pi i / m}$.

Proof. Set $\chi=\lambda+\bar{\lambda}$. First we show that $\chi$ is faithful. Let $L=\{\chi(g) \mid 1 \neq$ $g \in G\}$. Then $L=\{2 \cos (2 \pi r / m) \quad \mid 1 \leq r \leq(m-1) / 2\}$. If there exists $1 \leq r \leq(m-1) / 2$ such that $2 \cos (2 \pi r / m)=2$, then $2 \pi r / m=2 k \pi$ for some $k \in \mathbb{Z}$, a contradiction. Hence $\chi$ is faithful. Moreover,

$$
\begin{aligned}
\prod_{r=1}^{(m-1) / 2}\left(2-2 \cos \left(\frac{2 \pi r}{m}\right)\right) & =\prod_{r=1}^{(m-1) / 2} 4 \sin ^{2}\left(\frac{\pi r}{m}\right) \\
& =\prod_{r=1}^{(m-1) / 2} 2 \sin \left(\frac{\pi r}{m}\right) \prod_{r=1}^{(m-1) / 2} 2 \sin \left(\frac{\pi r}{m}\right) \\
& =\prod_{r=1}^{(m-1) / 2} 2 \sin \left(\frac{\pi r}{m}\right) \prod_{r=1}^{(m-1) / 2} 2 \sin \left(\frac{(m-r) \pi}{m}\right) \\
& =\prod_{r=1}^{(m-1) / 2} 2 \sin \left(\frac{\pi r}{m}\right) \prod_{j=(m+1) / 2}^{m-1} 2 \sin \left(\frac{\pi j}{m}\right) \\
& =\prod_{r=1}^{m-1} 2 \sin \left(\frac{\pi r}{m}\right)=m,
\end{aligned}
$$

(See [7, Lemma 3.1]). This shows that $\chi$ is a sharp character, as desired.

Lemma 3.4. Let $D_{2 m}$ be the dihedral group of order $2 m$, where $m$ is odd, and let $\chi$ be a faithful irreducible character of $D_{2 m}$ of degree 2. Then $\left(D_{2 m}, \chi\right)$ is a sharp pair of type $L=\left\{0, \omega^{r}+\omega^{-r} \mid 1 \leq r \leq(m-1) / 2\right\}$, where $\omega=e^{2 \pi i / m}$.

Proof. See the proof of Theorem 3.2 in [7].

Lemma 3.5. Let $G$ be a dihedral or generalized quaternion group of order $2 m$ where $m$ is even and let $\omega=e^{2 \pi i / m}$. Then 
(1) if $m / 4 \in \mathbb{Z}$ and $\psi$ is a faithful irreducible character of degree 2 , then $(G, \psi)$ is a sharp pair of type $L=\left\{-2,0, \omega^{r}+\omega^{-r} \quad \mid 1 \leq r \leq m / 2-1\right\}$;

(2) if $\psi$ is a faithful irreducible character of degree 2 and $\varepsilon$ is linear with cyclic kernel of order $m$, then $(G, \psi+\varepsilon)$ is a sharp pair of type

$$
L=\left\{-1,1+\omega^{r}+\omega^{-r} \mid 1 \leq r \leq m / 2-1\right\}
$$

Proof. Assume that $m=2 t$ for some integer $t$. If $G=D_{2 m}$, then

$$
G=\left\langle a, b \quad: \quad a^{m}=b^{2}=1, \quad b^{-1} a b=a^{-1}\right\rangle
$$

and it is known that Table 7 is the character table of $G$ where

$$
\{1\},\left\{a^{t}\right\},\left\{a^{r}, a^{-r}\right\} \text { for } 1 \leq r \leq t-1,\left\{a^{s} b \mid \text { s even }\right\},\left\{a^{s} b \mid s \text { odd }\right\}
$$

are the conjugacy classes of $G$. Similarly, if $G=Q_{2 m}$ is the generalized quaternion

\begin{tabular}{||c|cccrr||}
\hline$C l_{G}(g)$ & 1 & $a^{t}$ & $a^{r}(1 \leq r \leq t-1)$ & $b$ & $a b$ \\
$\left|C l_{G}(g)\right|$ & 1 & 1 & 2 & $m / 2$ & $m / 2$ \\
\hline$\chi_{1}$ & 1 & 1 & 1 & 1 & 1 \\
$\chi_{2}$ & 1 & 1 & 1 & -1 & -1 \\
$\chi_{3}$ & 1 & $(-1)^{t}$ & $(-1)^{r}$ & 1 & -1 \\
$\chi_{4}$ & 1 & $(-1)^{t}$ & $(-1)^{r}$ & -1 & 1 \\
$\psi_{j}$ & 2 & $2(-1)^{j}$ & $\omega^{j r}+\omega^{-j r}$ & 0 & 0 \\
$(1 \leq j \leq t-1)$ & & & & & \\
\hline
\end{tabular}

Table 7: Character table of $D_{2 m}(m$ even $)$

group of order $2 m$, then

$$
G=\left\langle a, b \quad: \quad a^{2 t}=1, \quad a^{t}=b^{2}, \quad b^{-1} a b=a^{-1}\right\rangle
$$

In this case

$$
\{1\},\left\{a^{t}\right\},\left\{a^{r}, a^{-r}\right\}
$$

for $1 \leq r \leq t-1$, and

$$
\left\{a^{2 r} b \mid 0 \leq r \leq t-1\right\},\left\{a^{2 r+1} b \mid 0 \leq r \leq t-1\right\}
$$


are the conjugacy classes of $G$ and the character tables of the groups $Q_{4 t}(t$ odd) and $Q_{4 t}$ ( $t$ even) are given in Table 8 and Table 9 , respectively; (see [6]). Now suppose that $G$ is one of the two groups $D_{2 m}$ or $Q_{2 m}$, ( $m$ even). We show that the irreducible character $\psi_{1}$ is a sharp character of $G$ of type

$$
L=\left\{-2,0, \omega^{r}+\omega^{-r} \mid 1 \leq r \leq m / 2-1\right\} .
$$

It is easy to see that $\psi_{1}$ is faithful and we have

$$
\begin{aligned}
\prod_{r=1}^{m / 2-1}\left(2-2 \cos \left(\frac{2 \pi r}{m}\right)\right) & =\prod_{r=1}^{m / 2-1} 4 \sin ^{2}\left(\frac{\pi r}{m}\right) \\
& =\prod_{r=1}^{m / 2-1} 2 \sin \left(\frac{\pi r}{m}\right) \prod_{r=1}^{m / 2-1} 2 \sin \left(\frac{\pi r}{m}\right) \\
& =\prod_{r=1}^{m / 2-1} 2 \sin \left(\frac{\pi r}{m}\right) \prod_{r=1}^{m / 2-1} 2 \sin \left(\frac{(m-r) \pi}{m}\right) \\
& =\prod_{r=1}^{m / 2-1} 2 \sin \left(\frac{\pi r}{m}\right) \prod_{j=m / 2+1}^{m-1} 2 \sin \left(\frac{\pi j}{m}\right) \\
& =\frac{\prod_{r=1}^{m-1} 2 \sin \left(\frac{\pi r}{m}\right)}{2 \sin \left(\frac{\pi}{2}\right)}=\frac{m}{2}
\end{aligned}
$$

(See [7, Lemma 3.1]). Since for $r=m / 4$, we have $2 \cos \left(\frac{2 \pi r}{m}\right)=0$ it follows that $\prod_{l \in L\left(\psi_{1}\right)}(2-l)=2 m$ and hence $\psi_{1}$ is a sharp character of $G$ of type

$$
L=\left\{-2,0, \omega^{r}+\omega^{-r} \mid 1 \leq r \leq m / 2-1\right\} .
$$

\begin{tabular}{||c|rrcrr||}
\hline$C l_{G}(g)$ & 1 & $a^{t}$ & $a^{r}(1 \leq r \leq t-1)$ & $b$ & $a b$ \\
$\left|C l_{G}(g)\right|$ & 1 & 1 & 2 & $m / 2$ & $m / 2$ \\
\hline$\chi_{1}$ & 1 & 1 & 1 & 1 & 1 \\
$\chi_{2}$ & 1 & 1 & 1 & -1 & -1 \\
$\chi_{3}$ & 1 & -1 & $(-1)^{r}$ & $i$ & $-i$ \\
$\chi_{4}$ & 1 & -1 & $(-1)^{r}$ & $-i$ & $i$ \\
$\psi_{j}$ & 2 & $2(-1)^{j}$ & $\omega^{j r}+\omega^{-j r}$ & 0 & 0 \\
$(1 \leq j \leq t-1)$ & & & & & \\
\hline
\end{tabular}

Table 8: Character table of $Q_{4 t}(t$ odd $)$ 


\begin{tabular}{||c|cccrr||}
\hline$C l_{G}(g)$ & 1 & $a^{t}$ & $a^{r}(1 \leq r \leq t-1)$ & $b$ & $a b$ \\
$\left|C l_{G}(g)\right|$ & 1 & 1 & 2 & $m / 2$ & $m / 2$ \\
\hline$\chi_{1}$ & 1 & 1 & 1 & 1 & 1 \\
$\chi_{2}$ & 1 & 1 & 1 & -1 & -1 \\
$\chi_{3}$ & 1 & 1 & $(-1)^{r}$ & 1 & -1 \\
$\chi_{4}$ & 1 & 1 & $(-1)^{r}$ & -1 & 1 \\
$\psi_{j}$ & 2 & $2(-1)^{j}$ & $\omega^{j r}+\omega^{-j r}$ & 0 & 0 \\
$(1 \leq j \leq t-1)$ & & & & & \\
\hline
\end{tabular}

Table 9: Character table of $Q_{4 t}(t$ even $)$

Now we consider irreducible character $\psi_{j}$ where $2 \leq j \leq t-1$. Then one can see that $\psi_{j}$ is faithful if and only if $j$ is odd and $(j, m)=1$. In this case we have $L\left(\psi_{1}\right)=L\left(\psi_{j}\right)$ and the argument above shows that $\psi_{j}$ is a sharp character for $G$ of type $L=\left\{-2,0, \omega^{r}+\omega^{-r} \mid 1 \leq r \leq m / 2-1\right\}$.

Similarly, we can show that $\chi_{2}+\psi_{j}$, where $1 \leq j \leq t-1$ is odd and $(j, m)=1$, is a sharp character of type $L=\left\{-1,1+\omega^{r}+\omega^{-r} \mid 1 \leq r \leq m / 2-1\right\}$ for $G$.

For the remainder of this paper we put $\omega=e^{2 \pi i / m}$ and we assume that

(a) $L_{1}=\left\{\omega^{r} \quad \mid 1 \leq r \leq m-1\right\}$ such that $m \geq 3$;

(b) $L_{2}=\left\{\omega^{r}+\omega^{-r} \mid 1 \leq r \leq(m-1) / 2\right\}$ such that $m \geq 5$ is odd;

(c) $L_{3}=\left\{0, \omega^{r}+\omega^{-r} \mid 1 \leq r \leq(m-1) / 2\right\}$ such that $m \geq 5$ is odd;

(d) $L_{4}=\left\{-2,0, \omega^{r}+\omega^{-r} \mid 1 \leq r \leq m / 2-1\right\}$ such that $m \geqslant 8$ is even;

(e) $L_{5}=\left\{-1,1+\omega^{r}+\omega^{-r} \mid 1 \leq r \leq m / 2-1\right\}$ such that $m \geqslant 8$ is even;

(f) $L_{6}=\{-2,-1,0,1, \sqrt{2},-\sqrt{2}\}$;

(g) $L_{7}=\left\{-2,-1,0,1, \frac{1+\sqrt{5}}{2}, \frac{1-\sqrt{5}}{2}, \frac{-1+\sqrt{5}}{2}, \frac{-1-\sqrt{5}}{2}\right\}$; 
(h) $L_{8}=\left\{-1,0, \frac{1+\sqrt{5}}{2}, \frac{1-\sqrt{5}}{2}\right\}$.

Then we can see that $L_{i} \cap \mathbb{Z} \subseteq\{-2,-1,0,1,2\}$ for $1 \leq i \leq 5$. In the next lemmas for cyclic groups, dihedral groups, and generalized quaternion groups we determine $L_{i} \cap \mathbb{Z}$ whenever $(G, \chi)$ is $L_{i}$-sharp.

Lemma 3.6. Let $G$ be a cyclic group of order $m$ and $(G, \chi)$ be $L$-sharp with $L=L_{1}$ or $L=L_{2}$, then $L \cap \mathbb{Z} \subseteq\{-1\}$.

Proof. First let $L=L_{1}$. Since $L_{1}$ is the set of $m$ th roots of unity (distinct from 1 ), it is clear that $L_{1} \cap \mathbb{Z} \subseteq\{-1\}$.

Now suppose that $L=L_{2}$. Since $m$ is odd, it is easy to see that $-2,0,1,2 \notin L_{2}$. Moreover, $\omega^{r}+\omega^{-r}=-1$ if and only if $m / 3 \in \mathbb{Z}$. So $L_{2} \cap \mathbb{Z} \subseteq\{-1\}$.

Lemma 3.7. Let $G$ be a dihedral group of order $2 m,(m \geq 5$ odd $)$ and $(G, \chi)$ be $L$-sharp with $L=L_{3}$, then $\{0\} \subseteq L \cap \mathbb{Z} \subseteq\{-1,0\}$.

Proof. Since $0 \in L_{3} \cap \mathbb{Z}$, it suffices to show that $-2,1,2 \notin L_{3}$. If $-2,1 \in L_{3}$, then we obtain a contradiction under assumption $m \geq 5$ is odd. Moreover, $r \leq(m-1) / 2$ shows that $2 \notin L_{3}$. Note that $\omega^{r}+\omega^{-r}=-1$ if and only if $m / 3$ is an integer. Therefore if $m / 3$ is integer, then $L \cap \mathbb{Z}=\{-1,0\}$.

Lemma 3.8. Let $G$ be a dihedral or generalized quaternion group of order $2 \mathrm{~m}$, $\left(m \geq 8\right.$ even) and $(G, \chi)$ be $L$-sharp with $L=L_{4}$, then $L \cap \mathbb{Z}=\{-2,0\}$ or $L \cap \mathbb{Z}=$ $\{-2,-1,0,1\}$.

Proof. Obviously, $-2,0 \in L_{4}$. Since $r \leq m / 2-1$, we have $2 \notin L_{4}$. It is easy to see that $-1,1 \in L_{4}$ if and only if $m / 3$ is an integer. Hence $L_{4} \cap \mathbb{Z}=\{-2,0\}$ or $L_{4} \cap \mathbb{Z}=\{-2,-1,0,1\}$.

Lemma 3.9. Let $G$ be a dihedral or generalized quaternion group of order $2 m,(m \geq 8$ even $)$ and $(G, \chi)$ be L-sharp with $L=L_{5}$. Then $L \cap \mathbb{Z} \in$ $\{\{-1\},\{-1,1\},\{-1,0,2\},\{-1,0,1,2\}\}$.

Proof. It follows from the assumption that $-1 \in L_{5}$ and $-2 \notin L_{5}$. Since $m$ is even, we have $0,2 \in L_{5}$ if and only if $m / 3$ is an integer. Moreover, $1 \in L_{5}$ if and only if $r=m / 4$. Therefore $L \cap \mathbb{Z}$ is equal to $\{-1\},\{-1,1\},\{-1,0,2\}$ or $\{-1,0,1,2\}$. 
Theorem 3.10. Suppose $(G, \chi)$ is L-sharp and normalized such that $L$ contains at least an irrational value. If $L \subseteq \mathbb{R}$ and

$$
L \cap \mathbb{Z} \in\{\emptyset,\{-1\},\{-1,1\},\{-1,0,2\},\{-1,0,1,2\}\},
$$

then $(\chi, \chi)_{G}=2$. Otherwise $(\chi, \chi)_{G}=1$.

Proof. First suppose that $L \subseteq \mathbb{R}$ and

$$
L \cap \mathbb{Z} \in\{\emptyset,\{-1\},\{-1,1\},\{-1,0,2\},\{-1,0,1,2\}\} .
$$

Then it follows from Theorem 3.1 and Lemmas 3.6, 3.7, 3.8, and 3.9 that

(1) either $G$ is a cyclic group of odd order and $\chi$ is the sum of two complex conjugate linear characters of $G$;

(2) or $G$ is a dihedral or generalized quaternion group of order $2 m$ ( $m \geq 8$ even) and $\chi$ is the sum of an irreducible character of degree 2 and a linear character with cyclic kernel of order $m$.

So in each of these two cases we have $(\chi, \chi)_{G}=2$.

Now we consider the other cases as follows.

(i) If $L \nsubseteq \mathbb{R}$, then by Theorem 3.1 and Lemmas 3.2, 3.3, 3.4, and 3.5, $G$ is a cyclic group and $\chi$ is linear.

(ii) If $L \subseteq \mathbb{R}$ and $L \cap \mathbb{Z} \notin\{\emptyset,\{-1\},\{-1,1\},\{-1,0,2\},\{-1,0,1,2\}\}$, then by Theorem 3.1 and Lemmas 3.6, 3.7, 3.8, and 3.9, we have one of the following cases:

(a) $G$ is dihedral of order $2 m$, where $m \geq 5$ is odd, and $\chi$ is irreducible of degree 2 .

(b) $G$ is dihedral or generalized quaternion of order $2 m$, where $m \geq 8$ is even, and $\chi$ is irreducible of degree 2 .

(c) $G$ is isomorphic to the binary octahedral group and $\chi$ is irreducible sharp character of degree 2 and type $L=L_{6}$.

(d) $G$ is isomorphic to $S L(2,5)$ and $\chi$ is irreducible sharp character of degree 2 and type $L=L_{7}$. 
(e) $G$ is isomorphic to $A_{5}$ and $\chi$ is irreducible sharp character of degree 3 and type $L=L_{8}$.

As we see in the cases $(i)$ and $(i i)$ above, the sharp character $\chi$ is irreducible and so we have $(\chi, \chi)_{G}=1$.

Now we are ready to state and prove the main result of this section.

Theorem 3.11. If $(G, \chi)$ is L-sharp and normalized with $|L| \geq 2$ and $L$ contains at least an irrational value, then $(\chi, \chi)_{G}$ is uniquely determined by $L$.

Proof. It follows from Theorem 3.10 that if $(G, \chi)$ is $L$-sharp with $|L| \geq 2$ and $L$ contains at least an irrational value, then $(\chi, \chi)_{G} \in\{1,2\}$ and it can be uniquely determined by $L$.

Remark 3.12. The example below shows that the pair $(G, \chi)$ in Theorem 3.11 must be normalized as a necessary condition.

Example 3.13. Let $G$ be the dihedral group $D_{16}$. Then $\chi=\chi_{3}+2 \chi_{1}$ is a sharp character of type $\{2,0, \sqrt{2}+2,-\sqrt{2}+2\}$; see Table 10 . On the other hand, $\theta=$ $\chi_{2}+\chi_{3}+\chi_{1}$ is also a sharp character of type $\{2,0, \sqrt{2}+2,-\sqrt{2}+2\}$. But we have $(\chi, \chi)_{G} \neq(\theta, \theta)_{G}$.

\begin{tabular}{c|rrrrrrr}
$\left|C l_{G}(g)\right|$ & 1 & 4 & 2 & 2 & 1 & 4 & 2 \\
\hline$\chi_{1}$ & 1 & 1 & 1 & 1 & 1 & 1 & 1 \\
$\chi_{2}$ & 1 & -1 & 1 & 1 & 1 & -1 & 1 \\
$\chi_{3}$ & 2 & 0 & $\sqrt{2}$ & 0 & -2 & 0 & $-\sqrt{2}$
\end{tabular}

Table 10: Sharp characters $\chi=\chi_{3}+2 \chi_{1}$ and $\theta=\chi_{2}+\chi_{3}+\chi_{1}$

\section{References}

[1] D. Alvis and S. Nozawa, Sharp characters with irrational values, J. Math. Soc. Japan, 48(3) (1996) 567-591.

[2] H. F. Blichfeldt, A theorem concerning the invariants of linear homogeneous groups, with some applications to substitution-groups, Trans. Amer. Math. Soc. 5 (1904) 461-466. 
[3] P. J. Cameron and M. Kiyota, Sharp characters of finite groups, J. Algebra, 115(1) (1988) 125-143.

[4] T. Ito and M. Kiyota, Sharp permutation groups, J. Math. Soc. Japan 33 (1981) 435-444.

[5] The GAP Group, GAP - Groups, Algorithms, and Programming, Version 4.8.8; 2017. (http://www.gap-system.org)

[6] G. James and M. Liebeck, Representations and Characters of Groups, Cambridge Mathematical Textbooks, Cambridge, 1995.

[7] E. Poimenidou and A. Cottrell, Total characters of dihedral groups and sharpness, Missouri J. Math Sci. 12(1) (2000) 12-25. 